\title{
On Some Identities and Generating Functions for Mersenne Numbers and Polynomials
}

\author{
Ali Boussayoud ${ }^{1}$, Mourad Chelgham ${ }^{2, *}$, Souhila Boughaba ${ }^{3}$ \\ ${ }^{1}$ LMAM Laboratory and Department of Mathematics, Mohamed Seddik Ben Yahia University, Jijel, Algeria \\ ${ }^{2}$ LMAM Laboratory and Departement of Mathematics, Freres Mentouri Constantine1 University, Algeria \\ ${ }^{3}$ Department of Mathematics, Mohamed Seddik Ben Yahia University, Jijel, Algeri \\ *Corresponding author: chelghamm@yahoo.fr
}

Received January 13, 2018; Revised March 07, 2018; Accepted June 20, 2018

\begin{abstract}
In this paper, we introduce a new operator in order to derive some properties of homogeneous symmetric functions. By making use of the proposed operator, we give some new generating functions for Mersenne numbers, Mersenne numbers and product of sequences and Chebychev polynomials of second kind.
\end{abstract}

Keywords: Mersenne numbers, Generating functions, Symmetric functions

2010 Mathematics Subject Classification. Primary 05E05; Secondary 11B39.

Cite This Article: Ali Boussayoud, Mourad Chelgham, and Souhila Boughaba, "On Some Identities and Generating Functions for Mersenne Numbers and Polynomials." Turkish Journal of Analysis and Number Theory, vol. 6, no. 3 (2018): 93-97. doi: 10.12691/tjant-6-3-5.

\section{Introduction}

In this paper we consider one of the sequences of positive integers satisfying a recurrence relation and we give some well-known identities for this type of sequences [9]. One of the sequences of positive integers (also defined recursively) that have been studied over several years is the well-known Fibonacci (and Lucas) sequence. Many papers are dedicated to Fibonacci sequence, such as the works of Hoggatt in [10], and Koshy in [11], among others. Others sequences satisfying a second-order recurrence relations are the main topic of the research for several authors, such as the studies of the sequences $\left\{J_{n}\right\}_{n \in \mathrm{N}}$ and $\left\{j_{n}\right\}_{n \in \mathrm{N}}$ of Jacobsthal and Jacobsthal-Lucas numbers, respectively.

In this paper we do not have such kind of generalization, but we will follow closely some of these studies. About the Mersenne sequence, also some studies about this sequence have been published, such as the work of Koshy [12], where the authors investigate some divisibility properties of Catalan numbers with Mersenne numbers Mk as their subscripts, developing their work in [12]. In number theory, recall that a Mersenne number of order $n$, denoted by $\mathrm{Mn}$, is a number of the form $2^{n}-1$, where $n$ is a nonnegative number. This identity is called as the Binet formula for Mersenne sequence and it comes from the fact that the Mersenne numbers can also be defined recursively by

$$
M_{n+1}=2 M_{n}+1,
$$

with initial conditions $M_{0}=0, M_{1}=1$. Since this recurrence is inhomogeneous, substituting $n$ by $n+1$, we obtain the new form

$$
M_{n+2}=2 M_{n+1}+1,
$$

Subtracting (1.1) to (1.2), we have that

$$
M_{n+2}-M_{n+1}=2 M_{n+1}+1-\left(2 M_{n}+1\right)
$$

and then

$$
M_{n+2}=3 M_{n+1}-2 M_{n},
$$

other form for the recurrence relation of Mersenne sequence, with initial conditions $M_{0}=0, M_{1}=1$. The roots of the respective characteristic equation

$$
r^{2}-3 r+2=0
$$

are

$$
r_{1}=1 \text { and } r_{2}=2,
$$

and we easily get the Binet formula

$$
M_{n}=2^{n}-1 .
$$

The main purpose of this paper is to present some results involving the Mersenne numbers using define a new useful operator denoted by $\delta_{p_{1} p_{2}}^{k}$ for which we can formulate, extend and prove new results based on our previous ones $[1,3,4]$. In order to determine generating functions of the product of Mersenne numbers and Chebychev polynomials of first and second kind, we combine between our indicated past techniques and these presented polishing approaches. 


\section{Definitions and Some Properties}

In order to render the work self-contained we give the necessary preliminaries tools; we recall some definitions and results.

Definition1. [6] Let $B$ and $P$ be any two alphabets. We define $S_{n}(B-P)$ by the following form

$$
\frac{\Pi_{p \varepsilon P}(1-p t)}{\Pi_{b \varepsilon B}(1-b t)}=\sum_{n=0}^{\infty} S_{n}(B-P) t^{n},
$$

with the condition $S_{n}(B-P)=0$ for $n<0$.

Equation (2.1) can be rewritten in the following form

$$
\sum_{n=0}^{\infty} S_{n}(B-P) t^{n}=\left(\sum_{n=0}^{\infty} S_{n}(B) t^{n}\right) \times\left(\sum_{n=0}^{\infty} S_{n}(-P) t^{n}\right),
$$

where

$$
S_{n}(B-P)=\sum_{j=0}^{n} S_{n-j}(-P) S_{j}(B) .
$$

We know that the polynomial whose roots are $P$ is written as

$$
S_{n}(x-P)=\sum_{j=0}^{n} S_{n-j}(-P) x^{n}, \text { with } \operatorname{card}(P)=n .
$$

On the other hand, if $B$ has cardinality equal to 1, i.e., $B=\{x\}$, then (2.1) can be rewritten as follows [6]:

$$
\begin{aligned}
& \sum_{n=0}^{\infty} S_{n}(x-P) t^{n}=\frac{\prod_{p \in P}(1-p t)}{(1-x t)} \\
& =1+\cdots+S_{n-1}(x-P) t^{n-1}+\frac{S_{n}(x-P)}{(1-x t)} t^{n},
\end{aligned}
$$

where $S_{n+k}(x-P)=x^{k} S_{n}(x-P)$ for all $k \geq 0$.

The summation is actually limited to a finite number of terms since $S-k(\cdot)=0$ for all $k>0$. In particular, we have

$$
\begin{aligned}
& \prod_{p \in P}(x-p)=S_{n}(x-P) \\
& =S_{0}(-P) x^{n}+S_{1}(-P) x^{n-1}+S_{2}(-P) x^{n-2}+\cdots,
\end{aligned}
$$

where $S_{k}(-B)$ are the coefficients of the polynomials $S_{n}(x-P)$ for $0 \leq k \leq n$. These coefficients are zero for $k>n$.

For example, if all $p \in P$ are equal, i.e., $P=n p$, then we have $S_{n}(x-n p)=(x-p)^{n}$

By choosing $P=1$ i.e., $P=\{\underbrace{1,1, \ldots 1}_{n}\}$, we obtain

$$
S_{k}(-n)=(-1)^{k}\left(\begin{array}{l}
n \\
k
\end{array}\right) \text { and } S_{k}(n)=\left(\begin{array}{c}
n+k-1 \\
k
\end{array}\right)
$$

By combining (2.2) and (2.3), we obtain the following expression

$$
\begin{aligned}
S_{n}(B-n x)= & S_{n}(B)-\left(\begin{array}{l}
n \\
1
\end{array}\right) S_{n-1}(B) x \\
& +\left(\begin{array}{l}
n \\
2
\end{array}\right) S_{n-2}(B) x^{2}-\cdots+(-1)^{n}\left(\begin{array}{l}
n \\
n
\end{array}\right) x^{n} .
\end{aligned}
$$

Definition 2. [4] Given a function $f$ on $\mathbf{R}^{n}$, the divided difference operator is defined as follows

$$
\partial_{p_{i} p_{i+1}}(f)=\frac{\left[\begin{array}{l}
f\left(p_{1}, \cdots, p_{i}, p_{i+1}, \cdots p_{n}\right) \\
-f\left(p_{1}, \cdots p_{i-1}, p_{i+1,} p_{i}, p_{i+2} \cdots p_{n}\right)
\end{array}\right]}{p_{i}-p_{i+1}} .
$$

Definition 3. The symmetrizing operator $\delta_{e_{1} e_{2}}^{k}$ is defined by

$$
\delta_{p_{1} p_{2}}^{k}(g)=\frac{p_{1}^{k} g\left(p_{1}\right)-p_{2}^{k} g\left(p_{2}\right)}{p_{1}-p_{2}} \text { for all } k \in \mathrm{N} .
$$

Proposition 1. [5] Let $P=\left\{p_{1}, p_{2}\right\}$ an alphabet, we define the operator $\delta_{e_{1} e_{2}}^{k}$ as follows

$$
\delta_{p_{1} p_{2}}^{k} g\left(p_{1}\right)=S_{k-1}\left(p_{1}+p_{2}\right) g\left(p_{1}\right)+p_{2}^{k} \partial_{p_{1} p_{2}} g\left(p_{1}\right),
$$

for all $k \in \mathrm{N}$.

\section{On the Generating Functions}

In our main result, we will combine all these results in a unified way such that they can be considered as a special case of the following Theorem.

Theorem 1. Let $A$ and $P$ be two alphabets, respectively, $\left\{a_{1}, a_{2}\right\}$ and $\left\{b_{1}, b_{2}\right\}$, then we have

$$
\begin{aligned}
& \sum_{n=0}^{\infty} S_{n}(A) S_{n+k-1}(P) z^{n} \\
& =\frac{\left(\begin{array}{l}
\left.S_{k-1}\left(p_{1}+p_{2}\right)-\left(a_{1}+a_{2}\right) \delta_{p_{1} p_{2}}^{k}\left(p_{2}\right) z\right) \\
-a_{1} a_{2} p_{1} p_{2} \delta_{p_{1} p_{2}}\left(p_{2}^{k-1}\right) z^{2}
\end{array}\right)}{\left(\sum_{n=0}^{\infty} S_{n}(-A) p_{1}^{n} z^{n}\right)\left(\sum_{n=0}^{\infty} S_{n}(-A) p_{2}^{n} z^{n}\right)}
\end{aligned}
$$

for all $k \in \mathbb{N}$.

Proof By applying the operator $\partial_{p_{1} p_{2}}$ to the series $f\left(p_{1}\right)=\sum_{n=0}^{\infty} S_{n}(A) p_{1}^{n+k} z^{n}$, we have

$$
\begin{aligned}
& \partial_{p_{1} p_{2}}\left(\sum_{n=0}^{\infty} S_{n}(A) p_{1}^{n+k} z^{n}\right) \\
= & \frac{\sum_{n=0}^{\infty} S_{n}\left(a_{1}+a_{2}\right) p_{1}^{n+k} z^{n}-\sum_{n=0}^{\infty} S_{n}\left(a_{1}+a_{2}\right) p_{2}^{n+k} z^{n}}{p_{1}-p_{2}}
\end{aligned}
$$




$$
\begin{aligned}
& =\sum_{n=0}^{\infty} S_{n}\left(a_{1}+a_{2}\right)\left(\frac{p_{1}^{n+k}-p_{2}^{n+k}}{p_{1}-p_{2}}\right) z^{n} \\
& =\sum_{n=0}^{\infty} S_{n}(A) S_{n+k-1}(P) z^{n} .
\end{aligned}
$$

On the other hand,

$$
\begin{aligned}
& \partial_{p_{1} p_{2}}\left(\frac{p_{1}^{k}}{\sum_{n=0}^{\infty} S_{n}(-A) p_{1}^{n} z^{n}}\right) \\
& =\frac{\frac{p_{1}^{k}}{\sum_{n=0}^{\infty} S_{n}(-A) p_{1}^{n} z^{n}}-\frac{p_{2}^{k}}{\sum_{n=0}^{\infty} S_{n}(-A) p_{2}^{n} z^{n}}}{p_{1}-p_{2}} \\
& =\frac{p_{1}^{k} \sum_{n=0}^{\infty} S_{n}(-A) p_{2}^{n} z^{n}-p_{2}^{k} \sum_{n=0}^{\infty} S_{n}(-A) p_{1}^{n} z^{n}}{\left(p_{1}-p_{2}\right)\left(\sum_{n=0}^{\infty} S_{n}(-A) p_{1}^{n} z^{n}\right)\left(\sum_{n=0}^{\infty} S_{n}(-A) p_{2}^{n} z^{n}\right)} \\
& =\frac{\left[\begin{array}{l}
p_{1}^{k}-p_{2}^{k}-\left(a_{1}+a_{2}\right)\left(p_{1}^{k} p_{2}-p_{2}^{k} p_{1}\right) z \\
-a_{1} a_{2}\left(p_{2}^{k} p_{1}^{2}-p_{1}^{k} p_{2}^{2}\right) z^{2}
\end{array}\right]}{\left(p_{1}-p_{2}\right)\left(\sum_{n=0}^{\infty} S_{n}(-A) p_{1}^{n} z^{n}\right)\left(\sum_{n=0}^{\infty} S_{n}(-A) p_{2}^{n} z^{n}\right)} \\
& =\frac{\left[\begin{array}{l}
\frac{p_{1}^{k}-p_{2}^{k}}{p_{1}-p_{2}}-\left(a_{1}+a_{2}\right) p_{1} p_{2}\left(\frac{p_{1}^{k-1}-p_{2}^{k-1}}{p_{1}-p_{2}}\right) \\
-a_{1} a_{2} p_{1} p_{2}\left(\frac{p_{1} p_{2}^{k-1}-p_{2} p_{1}^{k-1}}{p_{1}-p_{2}}\right) z^{2}
\end{array}\right]}{\left(\sum_{n=0}^{\infty} S_{n}(-A) p_{1}^{n} z^{n}\right)\left(\sum_{n=0}^{\infty} S_{n}(-A) p_{2}^{n} z^{n}\right)} \\
& =\frac{\left[\begin{array}{l}
S_{k-1}\left(p_{1}+p_{2}\right)-\left(a_{1}+a_{2}\right) \delta_{p_{1} p_{2}}^{k}\left(p_{2}\right) z \\
-a_{1} a_{2} p_{1} p_{2} \delta_{p_{1} p_{2}}\left(p_{2}^{k-1}\right) z^{2}
\end{array}\right]}{\left(\sum_{n=0}^{\infty} S_{n}(-A) p_{1}^{n} z^{n}\right)\left(\sum_{n=0}^{\infty} S_{n}(-A) p_{2}^{n} z^{n}\right)} .
\end{aligned}
$$

Thus, this completes the proof.

We now derive new generating functions of the products of some well-known polynomials. Indeed, we consider Theorem 1 in order to derive Mersenne numbers and Tchebychev polynomials of second kind and the symmetric functions

- If $k=0$ and $A=\{1,0\}$ we deduce the following lemma

Lemma [1] Given an alphabet $P=\left\{p_{1}, p_{2}\right\}$, we have

$$
\sum_{n=0}^{\infty} S_{n-1}\left(p_{1}+p_{2}\right) z^{n}=\frac{z}{\left(1-p_{1} z\right)\left(1-p_{2} z\right)}
$$

Replacing $p_{2}$ by $\left(-p_{2}\right)$ in (3.2), we obtain

$$
\sum_{n=0}^{\infty} S_{n-1}\left(p_{1}+\left[-p_{2}\right]\right) z^{n}=\frac{z}{\left(1-p_{1} z\right)\left(1+p_{2} z\right)}
$$

Choosing $p_{1}$ and $p_{2}$ such that

$$
\left\{\begin{array}{l}
p_{1} p_{2}=-2 \\
p_{1}-p_{2}=3
\end{array}\right.
$$

and substituting in (3.3) we end up with

$$
\sum_{n=0}^{\infty} S_{n-1}\left(p_{1}+\left[-p_{2}\right]\right) z^{n}=\frac{z}{1-3 z+2 z^{2}}
$$

which represents a generating function for Mersenne numbers, such that $M_{n}=S_{n-1}\left(p_{1}+\left[-p_{2}\right]\right)$.

If $k=0, k=1$ and $A=\left\{a_{1}, a_{2}\right\}$ we deduce the following theorems

Theorem 2. [7] Given two alphabets $A=\left\{a_{1}, a_{2}\right\}$ and $P=\left\{p_{1}, p_{2}\right\}$ we have

$$
\begin{aligned}
& \sum_{n=0}^{\infty} S_{n}(A) S_{n-1}(P) z^{n} \\
& =\frac{\left(a_{1}+a_{2}\right) z-a_{1} a_{2}\left(p_{1}+p_{2}\right) z^{2}}{\left(\sum_{n=0}^{\infty} S_{n}(-A) p_{1}^{n} z^{n}\right)\left(\sum_{n=0}^{\infty} S_{n}(-A) p_{2}^{n} z^{n}\right)} .
\end{aligned}
$$

Theorem 3. [8] Given two alphabets $A=\left\{a_{1}, a_{2}\right\}$ and $P=\left\{p_{1}, p_{2}\right\}$ we have

$$
\begin{aligned}
& \sum_{n=0}^{\infty} S_{n}(A) S_{n}(P) z^{n} \\
& =\frac{1-a_{1} a_{2} p_{1} p_{2} z^{2}}{\left(\sum_{n=0}^{\infty} S_{n}(-A) p_{1}^{n} z^{n}\right)\left(\sum_{n=0}^{\infty} S_{n}(-A) p_{2}^{n} z^{n}\right)} .
\end{aligned}
$$

From (3.5) we can deduce

$$
\begin{aligned}
& \sum_{n=0}^{\infty} S_{n-1}(A) S_{n-1}(P) z^{n} \\
& =\frac{z-a_{1} a_{2} p_{1} p_{2} z^{3}}{\left(\sum_{n=0}^{\infty} S_{n}(-A) p_{1}^{n} z^{n}\right)\left(\sum_{n=0}^{\infty} S_{n}(-A) p_{2}^{n} z^{n}\right)} .
\end{aligned}
$$

Case 1: Replacing $p_{2}$ by $\left(-p_{2}\right)$ and $a_{2}$ by $\left(-a_{2}\right)$ in (3.4) and (3.6) yields

$$
\begin{aligned}
& \sum_{n=0}^{\infty} S_{n}\left(a_{1}+\left[-a_{2}\right]\right) S_{n-1}\left(p_{1}+\left[-p_{2}\right]\right) z^{n} \\
= & \frac{\left(a_{1}-a_{2}\right) z+a_{1} a_{2}\left(p_{1}-p_{2}\right) z^{2}}{\left(1-a_{1} p_{1} z\right)\left(1+a_{2} p_{1} z\right)\left(1+a_{1} p_{2} z\right)\left(1-a_{2} p_{2} z\right)} \\
= & \frac{z-a_{1} a_{2} p_{1} p_{2} z^{3}}{\left(1-a_{1} p_{1} z\right)\left(1+a_{2} p_{1} z\right)\left(1+a_{1} p_{2} z\right)\left(1-a_{2} p_{2} z\right)}
\end{aligned}
$$


This case consists of four related parts.

Firstly, the substitutions of

$$
\left\{\begin{array} { l } 
{ a _ { 1 } - a _ { 2 } = 1 , } \\
{ a _ { 1 } a _ { 2 } = 1 , }
\end{array} \text { and } \left\{\begin{array}{l}
p_{1}-p_{2}=3, \\
p_{1} p_{2}=-2,
\end{array}\right.\right.
$$

in (3.7) give

$$
\begin{aligned}
& \sum_{n=0}^{\infty} S_{n}\left(a_{1}+\left[-a_{2}\right]\right) S_{n-1}\left(p_{1}+\left[-p_{2}\right]\right) z^{n} \\
= & \frac{z+3 z^{2}}{1-3 z-3 z^{2}+6 z^{3}+4 z^{4}},
\end{aligned}
$$

which represents a new generating function for product of Fibonacci numbers with Mersenne numbers, such that $F_{n} M_{n}=S_{n}\left(a_{1}+\left[-a_{2}\right]\right) S_{n-1}\left(p_{1}+\left[-p_{2}\right]\right)$.

Secondly, the substitution of

$$
\left\{\begin{array} { l } 
{ a _ { 1 } - a _ { 2 } = 3 , } \\
{ a _ { 1 } a _ { 2 } = - 2 , }
\end{array} \text { and } \left\{\begin{array}{l}
p_{1}-p_{2}=3, \\
p_{1} p_{2}=-2,
\end{array}\right.\right.
$$

in (3.8) give

$$
\begin{aligned}
& \sum_{n=0}^{\infty} S_{n-1}\left(a_{1}+\left[-a_{2}\right]\right) S_{n-1}\left(p_{1}+\left[-p_{2}\right]\right) z^{n} \\
= & \frac{z-4 z^{3}}{1-9 z+28 z^{2}-36 z^{3}+16 z^{4}},
\end{aligned}
$$

which represents a new generating function for Mersenne numbers of second order, such that

$$
M_{n}^{2}=S_{n-1}\left(a_{1}+\left[-a_{2}\right]\right) S_{n-1}\left(p_{1}+\left[-p_{2}\right]\right) .
$$

Thirdly, the substitution of

$$
\left\{\begin{array} { l } 
{ a _ { 1 } - a _ { 2 } = 1 , } \\
{ a _ { 1 } a _ { 2 } = 2 , }
\end{array} \text { and } \left\{\begin{array}{l}
p_{1}-p_{2}=3, \\
p_{1} p_{2}=-2
\end{array}\right.\right.
$$

in (3.8) give

$$
\begin{aligned}
& \sum_{n=0}^{\infty} S_{n-1}\left(a_{1}+\left[-a_{2}\right]\right) S_{n-1}\left(p_{1}+\left[-p_{2}\right]\right) z^{n} \\
= & \frac{z+4 z^{3}}{1-3 z-8 z^{2}+12 z^{3}+4 z^{4}},
\end{aligned}
$$

which represents a new generating function for product of Jacobsthal numbers with Mersenne numbers, such that $J_{n} M_{n}=S_{n-1}\left(a_{1}+\left[-a_{2}\right]\right) S_{n-1}\left(p_{1}+\left[-p_{2}\right]\right)$.

Finally, the substitution of

$$
\left\{\begin{array} { l } 
{ a _ { 1 } - a _ { 2 } = 2 , } \\
{ a _ { 1 } a _ { 2 } = 1 , }
\end{array} \text { and } \left\{\begin{array}{l}
p_{1}-p_{2}=3, \\
p_{1} p_{2}=-2
\end{array}\right.\right.
$$

in (3.8) give

$$
\begin{aligned}
& \sum_{n=0}^{\infty} S_{n-1}\left(a_{1}+\left[-a_{2}\right]\right) S_{n-1}\left(p_{1}+\left[-p_{2}\right]\right) z^{n} \\
= & \frac{z+2 z^{3}}{1-6 z+3 z^{2}+12 z^{3}+4 z^{4}} .
\end{aligned}
$$

which represents a new generating function for product of Pell numbers with Mersenne numbers, such that

$$
P_{n} M_{n}=S_{n-1}\left(a_{1}+\left[-a_{2}\right]\right) S_{n-1}\left(p_{1}+\left[-p_{2}\right]\right) .
$$

Case 2: Replacing $p_{2}$ by $\left(-p_{2}\right)$ and $a_{1}$ by $2 a_{1}$ and $a_{2}$ by $\left(-2 a_{2}\right)$ in $(3.4)$ yields

$$
\begin{aligned}
& \sum_{n=0}^{\infty} S_{n}\left(2 a_{1}+\left[-2 a_{2}\right]\right) S_{n-1}\left(p_{1}+\left[-p_{2}\right]\right) z^{n} \\
= & \frac{2\left(a_{1}-a_{2}\right) z+4 a_{1} a_{2}\left(p_{1}-p_{2}\right) z^{2}}{\left(1-2 a_{1} p_{1} z\right)\left(1+2 a_{2} p_{1} z\right)\left(1+2 a_{1} p_{2} z\right)\left(1-2 a_{2} p_{2} z\right)}
\end{aligned}
$$

The substitution of

$$
\left\{\begin{array}{l}
p_{1}-p_{2}=3 \\
p_{1} p_{2}=-2, \\
4 a_{1} a_{2}=-1
\end{array}\right.
$$

in (3.9) and set for ease on notations $x=a_{1}-a_{2}$, we reach

$$
\begin{aligned}
& \sum_{n=0}^{\infty} S_{n}\left(2 a_{1}+\left[-2 a_{2}\right]\right) S_{n-1}\left(p_{1}+\left[-p_{2}\right]\right) z^{n}=\sum_{n=0}^{\infty} M_{n} U_{n}(x) z^{n} \\
= & \frac{2 x z-3 z^{2}}{1-6 x z+(5+8 x) z^{2}-12 x z^{3}+4 z^{4}}
\end{aligned}
$$

which corresponds to a new generating function for the combined Mersenne numbers and Tchebychev polynomials of the second kind.

Theorem 4. For $n \in \mathbb{N}$, the new generating function of the product of Mersenne numbers $M_{n}$ and Tchebychev polynomials of first kind is given by

$$
\sum_{n=0}^{\infty} M_{n} T_{n}(x) z^{n}=\frac{x z-3 z^{2}+2 x z^{3}}{1-6 x z+(5+8 x) z^{2}-12 x z^{3}+4 z^{4}} .
$$

Proof We see that

$$
\begin{aligned}
& \sum_{n=0}^{\infty} M_{n} T_{n}(x) z^{n} \\
= & \sum_{n=0}^{\infty} S_{n-1}\left(p_{1}+\left[-p_{2}\right]\right)\left(\begin{array}{l}
S_{n}\left(2 a_{1}+\left[-2 a_{2}\right]\right) \\
-x S_{n-1}\left(2 a_{1}+\left[-2 a_{2}\right]\right)
\end{array}\right) z^{n} \\
= & \sum_{n=0}^{\infty} S_{n-1}\left(p_{1}+\left[-p_{2}\right]\right) S_{n}\left(2 a_{1}+\left[-2 a_{2}\right]\right) z^{n} \\
& -x \sum_{n=0}^{\infty} S_{n-1}\left(p_{1}+\left[-p_{2}\right]\right) S_{n-1}\left(2 a_{1}+\left[-2 a_{2}\right]\right) z^{n} \\
= & \sum_{n=0}^{\infty} M_{n} U_{n}(x) z^{n} \\
& -\frac{x}{2\left(a_{1}+a_{2}\right)} \sum_{n=0}^{\infty} S_{n-1}\left(p_{1}+\left[-p_{2}\right]\right)\left(\left(2 a_{1}\right)^{n}-\left(-2 a_{2}\right)^{n}\right) z^{n} \\
= & \sum_{n=0}^{\infty} M_{n} U_{n}(x) z^{n} \\
& -\frac{x}{2\left(a_{1}+a_{2}\right)}\left(\begin{array}{l}
\sum_{n=0}^{\infty} S_{n-1}\left(p_{1}+\left[-p_{2}\right]\right)\left(2 a_{1} z\right)^{n} \\
-\sum_{n=0}^{\infty} S_{n-1}\left(p_{1}+\left[-p_{2}\right]\right)\left(-2 a_{2} z\right)^{n}
\end{array}\right) . \\
& \\
&
\end{aligned}
$$


On the other hand, we know that

$$
\sum_{n=0}^{\infty} S_{n-1}\left(p_{1}+\left[-p_{2}\right]\right) z^{n}=\frac{z}{1-3 z+2 z^{2}},
$$

from which it follows

$$
\begin{aligned}
& \sum_{n=0}^{\infty} M_{n} T_{n}(x) z^{n} \\
& =\frac{2 x z-3 z^{2}}{1-6 x z+(5+8 x) z^{2}-12 x z^{3}+4 z^{4}} \\
& -\frac{x}{2\left(a_{1}+a_{2}\right)}\left(\frac{2 a_{1} z}{1-6 a_{1} z+8 a_{1}^{2} z^{2}}+\frac{2 a_{2} z}{1+6 a_{2} z+8 a_{2}^{2} z^{2}}\right),
\end{aligned}
$$

Therefore

$$
\sum_{n=0}^{\infty} M_{n} T_{n}(x) z^{n}=\frac{x z-3 z^{2}+2 x z^{3}}{1-6 x z+(5+8 x) z^{2}-12 x z^{3}+4 z^{4}} .
$$

\section{Acknowledgements}

The authors would like to thank the anonymous referees for their valuable comments and suggestions.

\section{References}

[1] A. Boussayoud, M. Kerada, N. Harrouche, On the k-Lucas numbers and Lucas Polynomials, Turkish Journal of Analysis and Number.5(3) 121-125, (2017)

[2] A. Boussayoud, M. Bolyer, M. Kerada, On Some Identities and Symmetric Functions for lucas and pell numbers, Electron. J. Math. Analysis Appl. 5(1), 202-207, (2017).

[3] A. Boussayoud, On some identities and generating functions for Pell-Lucas numbers, Online.J. Anal. Comb. 12 1-10, (2017).

[4] A. Boussayoud, N. Harrouche, Complete Symmetric Functions and $k$ - Fibonacci Numbers, Commun. Appl. Anal. 20, 457-467, (2016).

[5] A. Boussayoud, M. Boulyer, M. Kerada, A simple and accurate method for determination of some generalized sequence of numbers, Int. J. Pure Appl. Math.108, 503-511, (2016)

[6] A. Boussayoud, A. Abderrezzak, M. Kerada, Some applications of symmetric functions, Integers. 15, A\#48, 1-7, (2015).

[7] A. Boussayoud, M. Kerada, R. Sahali , W. Rouibah, Some Applications on Generating Functions, J. Concr. Appl. Math. 12, 321-330, (2014).

[8] A. Boussayoud, M. Kerada, Symmetric and Generating Functions, Int. Electron. J. Pure Appl. Math. 7, 195-203(2014).

[9] P. Catarino, H. Campos, P. Vasco, On the Mersenne sequence, Ann. Math. Inform.46, 37-53, (2016).

[10] V. E, Hoggatt, Fibonacci and Lucas Numbers. A publication of the Fibonacci Association. University of Santa Clara, Santa Clara, Houghton Mifflin Company, 1969.

[11] T. Koshy, Fibonacci and Lucas Numbers with Applications, John Wiley, New York, 2001.

[12] T. Koshy, Z. Gao, Catalan numbers with Mersenne subscripts, Math. Sci. 38, 86-91 (2013). 